\title{
Three-dimensional quantification of capillary networks in healthy and cancerous tissues of two mice
}

\author{
Sabrina Lang ${ }^{1}$, Bert Müller ${ }^{1, *}$, Marco D. Dominietto ${ }^{2}$, Philippe C. Cattin ${ }^{3}$, Irene Zanette ${ }^{4}$, \\ Timm Weitkamp ${ }^{4,5}$, and Simone E. Hieber ${ }^{1, *}$
}

${ }^{1}$ Biomaterials Science Center, University of Basel, clo University Hospital, 4031 Basel, Switzerland

${ }^{2}$ Institute for Biomedical Engineering, ETH Zürich, 8093 Zürich, Switzerland

${ }^{3}$ Medical Image Analysis Center, University of Basel, c/o University Hospital, 4031 Basel, Switzerland

${ }^{4}$ European Synchrotron Radiation Facility, 38042 Grenoble, France

${ }^{5}$ Synchrotron Soleil, 91190 Gif-sur-Yvette, France

*Corresponding Authors

Email addresses: bert.mueller@unibas.ch (B. Müller), weitkamp@synchrotron-soleil.fr (T.

Weitkamp), simone.hieber@unibas.ch (S. Hieber).

\begin{abstract}
A key issue in developing strategies against diseases such as cancer is the analysis of the vessel tree in comparison to the healthy one. In the search for parameters that might be characteristic for tumor capillaries we study the vascularization in mice for cancerous and healthy tissues using synchrotron radiation-based micro computed tomography in absorption and phase contrast mode. Our investigations are based on absorption tomograms of casted healthy and cancerous tissues as well as a phase tomogram of a fixated tumor. We demonstrate how the voxel-based tomography data can be vectorized to assess the capillary networks quantitatively. The processing includes segmentation, skeletonization, and vectorization to finally extract the vessel parameters. The mean diameter of capillaries in healthy and cancerous tissues corresponds to $(8.0 \pm 1.1) \mu \mathrm{m}$ and $(3.9 \pm 1.1) \mu \mathrm{m}$, respectively. Further evaluated parameters show marginal or no differences between capillaries in healthy and cancerous tissues, namely fractal dimension $2.3 \pm 0.3$ vs. $2.3 \pm 0.2$, tortuosity (SOAM) $0.18 \mathrm{rad} / \mu \mathrm{m}$ vs. $0.24 \mathrm{rad} / \mu \mathrm{m}$ and vessel length $20 \mu \mathrm{m}$ vs. $17 \mu \mathrm{m}$. The bifurcation angles exhibit a narrow distribution around 116 degrees. Furthermore, we show that phase tomography is a powerful alternative to absorption tomography of casts for the vessel visualization omitting any invasive specimen preparation procedure.
\end{abstract}

Keywords:

Tumor

Tortuosity

$\mathrm{X}$-ray phase contrast

Synchrotron radiation

Vessel segmentation

Vectorization

Bifurcation

Vessel diameter

Automatic characterization 


\section{Introduction}

The three-dimensional (3D) analysis of the vessel tree including smallest capillaries is one of the key issues in tissue characterization and cancer research. While in vivo methods such as magnetic resonance imaging (MRI) only provide the angiogenic network indirectly because of limited spatial resolution, highly complex ex vivo techniques such as synchrotron radiationbased micro computed tomography $(\mathrm{SR} \mu \mathrm{CT})$ allow the direct visualization of the capillaries (Müller et al., 2006b; Plouraboué et al., 2004). For these studies, however, the absorption contrast between the vessels and the surrounding soft tissues has to be improved to allow for vessel segmentation (Müller et al., 2008), which requires staining protocols (Müller et al., 2006a; Plouraboue et al., 2004), tissue embedding (Müller et al., 2006b) or corrosion casting (Krucker et al., 2006). These procedures only work for intact vessel walls. In advanced stages of tumor development, the vessels become leaky and the contrast agents, embedding compound and low viscosity resin for corrosion casting can penetrate through the damaged parts of the vessel walls. For these cases, phase contrast SR $\mu$ CT should be applied, since the achievable contrast for soft tissues is orders of magnitude higher than for the conventional absorption contrast mode. Hence the complicated tissue preparation procedures can be omitted at the expense of higher experimental efforts. Holotomography considers the distribution of the phase (and attenuation) in two-dimensional projection images, and then translates it into 3-D reconstructions. The images are acquired during a rotation of the specimen for several specimen-detector distances. Using holotomography the resolution is considerably improved, but limited by the detector to about $1 \mu \mathrm{m}$. The spatial resolution of many phase contrast techniques, however, prohibits the identification of the smallest capillaries. Therefore, further efforts have to be invested to extract the vessel tree with reasonable contrast and true micrometer resolution so that the entire network of vessels within a reasonably large part of the tumor can be segmented. For the phase contrast tomography data the vessels are difficult to segment using intensity-based approaches such as choosing an appropriate threshold. Therefore, we propose the segmentation of the vessel tree using a tool that detects vessels by means of their characteristic geometry (tubular structure), rather than by a range of gray values. The feature-based method Frangi filter (Frangi et al., 1998) is a well-established tool for the segmentation of vessels (Lesage et al., 2009).

The limited photon statistics, always present in SR $\mu \mathrm{CT}$ datasets (Beckmann et al., 2008), commonly impedes the segmentation of continuous vessel networks from arteries via capillaries toward veins. The intact passage through this sequence of different vessel types, however, is a well-established fact and, therefore, a vital prerequisite for simulating tumor formation and growth. A direct comparison of these simulations with experimental highresolution data by means of the vessel tree parameters requires not only the segmentation itself but also includes the skeletonization (Bullitt et al., 2003b; Wild et al., 2000) and vectorization of the vessel tree from the $\mathrm{SR} \mu \mathrm{CT}$ data.

Once the vessel tree has been successfully extracted, one can analyze the numerous parameters that characterize the vascular structure of healthy and cancerous tissues (Brey et al., 2002; Bullitt et al., 2003a; Bullitt et al., 2003b; Folarin et al., 2010; Heinzer et al., 2006; Rijken et al., 1995; Wu et al., 2008). So far, the tortuosity of the vessel tree has been reported to be a key indicator to identify diseased tissue (Bullitt et al., 2003b).

The experimental parameters are necessary for the validation of tumor growth simulations that can reveal detailed insight of neo-vascularization (Lloyd et al., 2007). Such validated simulations are promising paths to substantially ameliorate anti-angiogenesis treatments against cancer (Folarin et al., 2010).

So far, it is still unclear how well the simulations of tumor growth describe the complex processes within the human body. In the search for parameters that might be characteristic for colon carcinoma induced cancerous vessels with respect to healthy ones, we have examined the murine vascular structure of tissues on the basis of SR $\mu \mathrm{CT}$ data. Corrosion casts of 
healthy and cancerous tissues represent a two-component, solid-state system, but also imply the presence of preparation artifacts, which, for example, inhibit the precise measurement of vessel diameters. Therefore, we have additionally measured tumor specimens using phase contrast SR $\mu \mathrm{CT}$. To the best of our knowledge, we are the first group to compare these data quantitatively with the ones of the conventional absorption contrast $\mathrm{SR} \mu \mathrm{CT}$.

High-resolution absorption and phase tomograms are processed to produce a vector-based representation of the vessel trees. In the present communication we show the in-depth comparison of capillary networks from healthy and diseased tissues exploring the vessel diameters, bifurcation densities (branching), angles between branching vessels, vessel/mean void distances, vessel lengths, tortuosity, fractal dimension, and vessel volume fraction with the aim to find the leading indicators for the formation of cancerous tissue. For example, Di Ieva et al. (2011) have shown how the fractal dimension, derived from two-dimensional images using the box-counting method, can be used to quantitatively characterize the microvasculature of normal and adenomatous pituitary tissues.

\section{Material and Methods}

\section{Tissue preparation}

Two balb/c nude mice (Charles River Laboratories, France) weighting $24 \mathrm{~g}$ to $27 \mathrm{~g}$ at an age of 10 weeks were used for the experiment. A suspension with $10^{6} \mathrm{C} 51$ tumor cells from murine colon carcinoma (Institute for Biomedical Engineering, ETH Zurich, Switzerland) was subcutaneously injected on the left flank of both mice (balb/c - Charles River Laboratories, France) in strict adherence to the Swiss law for animal protection.

Mice were euthanized using a solution of ketamine/xylazine at Day 7 (mouse A) and Day 14 (mouse B) following tumor inoculation.

The tumor $\left(3 \mathrm{~mm}^{3}\right.$ in size) from mouse A was extracted and fixed in $5 \%$ para-formaldehyde solution (PFA). The tumor was then transferred into the formalin-filled polymer container just before the SR $\mu \mathrm{CT}$ measurements in phase contrast mode.

The tumor from mouse B was casted using a polyurethane-based material according to the procedure described by Krucker et al. (2006). To obtain the cast, the polymer PUii4 together with a hardener was injected into the anesthetized mouse via the left ventricle of the heart. After curing the surrounding soft and hard tissues were corroded using formic acid. This cast was fragmented to harvest the tumor vessel tree with a size of about $(10 \mathrm{~mm})^{3}$ from the left flank and the casted vessels from healthy tissue $(45 \mathrm{~mm})^{3}$ in size from the right flank. The casts were visualized using $\mathrm{SR} \mu \mathrm{CT}$ in absorption contrast mode.

\section{Data acquisition}

Phase contrast-based SR $\mu \mathrm{CT}$ was carried out using propagation-based technique termed holotomography (Cloetens et al., 1999; Zabler et al., 2005) and conducted at the beamline ID 19 (ESRF, Grenoble, France) (Weitkamp et al., 2010). Holotomography relies on phase contrast generated by the propagation of $\mathrm{X}$ rays in free space between tumor and detector. The additional information (with respect to the conventional absorption imaging) needed to derive the phase was obtained by recording images at different propagation distances. The spatial resolution was usually limited by the detector pixel size. The detector consisted of a $19-\mu \mathrm{m}-$ thick gadolinium gallium garnet scintillator, microscope optics with $10 \times$ overall magnification, and a CCD camera (ESRF FReLoN model 230-42, 2048×2048 pixels of $15 \mu \mathrm{m}$ size), resulting in an effective pixel size of $1.5 \mu \mathrm{m}$. Using a photon energy of $17.6 \mathrm{keV}$, filtered from the undulator spectrum by means of a $\mathrm{Ru} / \mathrm{B}_{4} \mathrm{C}$-multilayer with $4 \mathrm{~nm}$ period, and an exposure time of $0.3 \mathrm{~s}$ per radiograph, 800 radiographs for equidistant viewing angles between 0 and $180^{\circ}$ were obtained for each of the three specimen-detector distances 60,120 and $360 \mathrm{~mm}$. The holotomography data required a phase retrieval procedure prior to tomographic reconstruction. In the following, we shall refer to tomographic reconstruction of phase-retrieved data as 'phase tomography'. The phase retrieval algorithm, implemented in GNU Octave version 2.1.73, was 
a mixed approach between the contrast transfer function (CTF) and the transport of intensity equation (TIE) (Guigay et al., 2007; Langer et al., 2008; Langer et al., 2010).

Local tomography data of the cast from the cancerous tissue were recorded at the TOMCAT beamline (Swiss Light Source (SLS) at Paul Scherrer Institut, Villigen, Switzerland) (Stampanoni et al., 2006) using a photon energy of $15 \mathrm{keV}$ and a pixel size of $2 \times 0.74 \mu \mathrm{m}$. The exposure time for each of the 1500 radiographs for equidistant rotation angles between 0 and $180^{\circ}$ was set to $0.7 \mathrm{~s}$. The 4 Mpixel detector covered only around $15 \%$ of the cast's projection.

The tomography data of the cast from the healthy tissue were acquired at the beamline BW 2 (HASYLAB at DESY, Hamburg, Germany). The Helmholtz Center Geesthacht, Germany operated the tomography setup (Beckmann, 2002). Using a photon energy of $10 \mathrm{keV}$, a pixel size of $1.98 \mu \mathrm{m}$ and an exposure time of $5.85 \mathrm{~s}, 1440$ radiographs equidistantly acquired along $360^{\circ}$ with an asymmetric rotation axis (Müller et al., 2007) were combined to 720 radiographs used for data reconstruction. The spatial resolution determined from the $10 \%$ value of the modulation transfer function corresponded to $4.7 \mu \mathrm{m}$ (Müller et al., 2002a). The $\mathrm{X}$ rays were converted to visible light by means of $\mathrm{CdWO}_{4}$ scintillator with a thickness of $200 \mu \mathrm{m}$.

The tomographic reconstructions were performed using the software tools available at the respective beamlines based on the conventional filtered back-projection algorithm (Kak and Slaney, 1988).

\section{Image analysis}

The image analysis of the vascular structure was conducted for the absorption tomograms of the casted healthy and cancerous tissues as well as for the phase tomogram of the fixated tumor. To evaluate the local differences in the vessel parameters, two regions of interests were selected in each tomography dataset. One region of interest $\left(\mathrm{ROI}_{1}\right)$ with a size of $180 \times 180 \times 180$ voxels and a larger one $\left(\mathrm{ROI}_{2}\right)$ with a size of $590 \times 590 \times 500$ voxels were chosen, such that they were filled with vascular structures.

The tomography data were segmented using an implementation of the Frangi filter (see below, section 'Segmentation') in Matlab 7.10.9 R2010a (Simulink, The MathWorks, Inc., USA) by Kroon (2009) and skeletonized using a software tool implemented in MS Visual C++ 2010 Express V.4.0.30319RTMRel (Microsoft Corp., Redmond, WA, USA) presented by (Cornea et al., 2005). For the vectorization we developed a program in Matlab 7.10.9 R2010a. VG Studio Max 2.0 (Volume Graphics, Heidelberg, Germany) served for generating the 3D representations.

\section{Segmentation}

In the case of the corrosion cast it was sufficient to set an appropriate intensity-based threshold for the segmentation. The threshold was defined in the absorption histogram by the intersection point of two Gaussians related to the cast and the surrounding air (Müller et al. 2002b, Lang et al., 2012). The number of voxels representing air was almost equal to the one representing the cast.

In the phase contrast dataset, however, the intensity values of the vessel strongly overlapped with the values of other structures. Therefore, a feature-based algorithm known as 'Frangi filter' was used for the vessel segmentation in the holotomograms. The Frangi filter is based on the eigenvalues $\lambda=\left(\lambda_{1}, \lambda_{2}, \lambda_{3}\right)$ of the 3D Hessian matrix (Sato et al., 1998) and provides the probability that voxel belongs to a vessel, e. g. its vesselness (Aharinejad et al., 1998; Frangi et al., 1998). In this context the shape of the vessel was assumed to be tubular (Collins et al., 2011; Zhou et al., 2006). The vesselness function of the scale $s$ is described by: 


$$
V_{0}(s)=\left\{\left(1-\exp \left(-\frac{R_{A}^{2}}{2 \alpha^{2}}\right)\right) \exp \left(-\frac{R_{B}^{2}}{2 \beta^{2}}\right)\left(1-\exp \left(-\frac{S^{2}}{2 \gamma^{2}}\right)\right) \quad \begin{array}{l}
\text { if } \lambda_{2}>0 \vee \lambda_{3}>0 \\
\text { otherwise }
\end{array}\right.
$$

The ratio $R_{\mathrm{A}}=\left|\lambda_{2}\right| /\left|\lambda_{3}\right|$ distinguishes between sheet- and tube-like structures, the ratio $R_{\mathrm{B}}=\left|\lambda_{1}\right| / \sqrt{\left|\lambda_{2} \lambda_{3}\right|}$ accounts for spherical structures and $S=|\lambda|$ for the second order structureness. For an ideal tubular structure, the eigenvalues fulfill the conditions $\left|\lambda_{1}\right| \approx 0,\left|\lambda_{1}\right|<<\left|\lambda_{2}\right|, \lambda_{2} \approx \lambda_{3}$ (Frangi et al., 1998; Sato et al., 1998). The constants $\alpha, \beta$, and $\gamma$ determine the sensitivity of the filter measures $R_{\mathrm{A}}, R_{\mathrm{B}}$, und $S$. The sign of eigenvalues $\lambda_{2}$ and $\lambda_{3}$ reflects whether dark vessels reside in a bright background or vice versa. Finally, the vesselness measure is the maximum value of the vesselness function within the range $s_{\min } \leq s \leq$ $s_{\max }$.

In the present study, the scale range for the tube-like structures was chosen from 1 to 6 voxels $(1.5$ to $9 \mu \mathrm{m})$. The histogram of the resulting vesselness measure revealed an exponential decay. The data were binarized setting a threshold at $1 \%$ of the highest frequency detected. The threshold value of $1 \%$ results from a trade-off between capturing blood vessels and avoiding detection of structures caused by noise. It was chosen manually by visual inspection. An increase of the threshold led to the segmentation of noisy structures, while a decrease reduced the volume of the segmented blood vessels.

\section{Vessel volume fraction and mean void distance}

The vessel volume fraction represents the ratio between the voxels belonging to a vessel and the total number of voxels. The vessel volume fraction was determined in the $3 \mathrm{D}$ dataset and in the three orthogonal 2D sections. Three-dimensional distance transforms of the datasets lead to the analysis of the void distance in 3D and its mean. The results were obtained using every other voxel of the discretized vessel to reduce misleading effects due to the voxel discretization of the vessels.

\section{Vectorization and bifurcation analysis}

The centerlines of the vessels were extracted using a skeletonization method based on voxel erosion (Cornea et al., 2005). A 3D connected component labeling algorithm was used to order the skeleton data by its number of object voxels. The neighboring voxels of each object were determined step by step from an arbitrary seed point. We neglected objects with a total size of less than 500 voxels and replaced connected line segments by one segment line if the vessel path remained the same, i. e. a straight line. The number of interrupted vessels was reduced by bridging gaps of less than 10 voxels in the vectorized tree. The correction mainly improved the analysis of the vessel length. Finally, the vessel segments were represented by sorted lists of centerline points. The data components of a vessel included $n$ positions of the vector points $r_{\mathrm{i}}$, the type of both endings $T_{1}$ and $T_{\mathrm{n}}$ (0: end-point, 1 : bifurcation point), its mean radius $R m$ with its standard deviation $R s$ and the vessel length $L$. The radii at the centerline points were derived from the distance transform of the dataset and averaged to the mean radius $R m$ leading to the mean diameter $D m=2 R m$. The vessel length $L$ was evaluated by the sum of all distances between the vector points. Figure 1 illustrates the meaning of the parameters.

The bifurcation density was described by two parameters, the relationship of the number of bifurcation points to the number of vessel segments and the one to the volume. The bifurcation angle represented the mean plane angle between the vector segments adjacent to the branching point (bifurcation points). 


\section{Tortuosity}

The tortuosity of the vessel tree was analyzed in terms of the distance metric (DM) and the sum-of-angle metrics (SOAM) (Bullitt et al., 2003b). Distance metric (DM) corresponds to the ratio of the vessel path length to the distance between endpoints and bifurcation points (Brey et al., 2002). Vessels deviating from a straight line showed a distance metric value larger than one. Since this approach could not distinguish between sinusoidal and C-curved vessels, Bullitt et al. (2003b) introduced the sum-of-angle metric. It was based on the ratio between the total curvature of segment and the vessel length, where the total curvature, the integral of the local curvature over the segment, was geometrically interpreted as a sum of the in-plane angle $\gamma_{I P}$ and the tortuous angle $\gamma_{T P}$ :

$$
\mathrm{SOAM}=\frac{\sum_{k=2}^{n-2}\left(\sqrt{\gamma_{I P, k}^{2}+\gamma_{T P, k}^{2}}\right)}{L}
$$

In case of co-linearity between the two vector points the tortuous angle was defined as zero. The SOAM yielded a value of zero for a straight line.

\section{Vessel diameter and length}

The mean vessel diameter was calculated from the vessel radii $R m$. The distribution of these radii follow Gaussians. Fitting the Gaussians using the Levenberg-Marquardt algorithm of the proFit software 6.2.4 (Quantum Soft, Ueticon am See, Switzerland), the mean values including their standard deviations were found. The vessel length is the mean of the vessel path lengths including inter-branch distances. In this manner, the lower limit of the mean vessel length was determined.

\section{Fractal dimension}

The fractal dimension was evaluated according to the box-counting algorithm (Mancardi et al., 2008; Mandelbrot, 1982) considering the 3D vessel segmented structure with an overlapped 3D grid. For a given size $s$ of the grid, it was possible to count the number of boxes $N(s)$ needed to cover the whole vessel structure. Iterating this procedure with different values of $s$, fractal dimension $F_{\mathrm{D}}$ was defined as:

$$
F_{D}=-\lim _{s \rightarrow 0} \frac{\log [N(s)]}{\log (s)}
$$

\section{Results}

\section{Phase contrast}

Considering the distribution of phase in the projection images, phase tomography allowed the vessels in the tumor to be identified without contrast enhancement procedures (see bright spots in Fig. 2). Most of the vessels were located in the outer region of the tumor.

\section{Quantification}

The quantitative data as described in the section above were determined for all specimens in the search for characteristic parameters (see Table 1).

\section{Vessel volume fraction and mean void distance}

The vessel volume fraction featured an anisotropic behavior in most of the selected regions (see Table 1) as given by the rather constant bifurcation angle of about $115^{\circ}$. Figure 3 also illustrates this observation in terms of the $3 \mathrm{D}$ distance transform (tumor cast, $\mathrm{ROI}_{1}$ ), where the vessels appear in black. The colored void distance differed in the orthogonal sections in its maximum and its distribution. The void region contains the voxels that are not associated with vessel. The largest void region was found in the green-color bordered section surrounded by rather dense vessels, whereas the vessels were distributed more uniformly in the other two 
sections. The mean void distance was inversely proportional to the vessel volume fraction in $\mathrm{ROI}_{2}$.

\section{Vectorization and bifurcation analysis}

Figure 4 shows the segmented vessel trees and their vectorization for $\mathrm{ROI}_{1}$, where the redcolored dots represent the bifurcation points. The bifurcation density with respect to the number of vessel segments was found to be $48 \%$ for the healthy cast, $28 \%$ for the tumor cast, and $23 \%$ for the phase contrast data set. This corresponds to volume density values for the bifurcations of $13,000 \mathrm{~mm}^{-3}$ in the healthy tissue, to $56,000 \mathrm{~mm}^{-3}$ in the cancerous cast, and to $30,000 \mathrm{~mm}^{-3}$ in the phase tomography data. The bifurcation angles exhibited a narrow distribution between $107^{\circ}$ and $119^{\circ}$ (see Table 1).

\section{Tortuosity}

Table 1 shows the tortuosity, the deviation from the straight centerline, in terms of DM and SOAM. The maximum values of the tortuosity based on the SOAM were found for the cancer cast while the healthy cast and the phase contrast led to similar results. Figure 5 illustrates the appearance of tortuosity in the healthy and cancerous vasculature. While the tumor featured a large variety of tortuous vessels, only a few capillaries appeared highly tortuous in the healthy tissue.

\section{Vessel diameter and length}

The mean vessel diameter ranged from 5 to $15 \mu \mathrm{m}$. For most of the analyzed datasets the healthy vessel cast exhibited the largest values for the diameter. Figure 6 shows the diameter distributions of the healthy and the cancerous cast where the profiles are clearly distinguishable. The determination of the vessel radius was rather robust against the choice of the segmentation threshold. Using holotomography the mean vessel radius increased by $4 \%$ when the threshold applied to the vesselness was four times higher and decreased by $5 \%$ when the threshold was halved. The reliability of the result was tested by evaluating 30 diameters ranging from 4 to $16 \mu \mathrm{m}$ manually and using the parameterized vessel tree. The mean capillary diameters of the vectorized data set differed about $8 \%$ from the value determined manually (absolute difference $0.6 \mu \mathrm{m}$ ).

The vessel length varied between 10 and $35 \mu \mathrm{m}$ where the phase contrast based dataset featured the smallest values for all ROIs.

\section{Fractal dimension}

The fractal dimension of the vessel trees extracted from the casts corresponded to $2.3 \pm 0.2$ for the cancerous tissue and $2.3 \pm 0.3$ for the healthy tissue. The result of phase contrast data was comparable $(2.2 \pm 0.6)$.

\section{Discussion}

The experiments were performed using absorption and phase contrast $\mathrm{SR} \mu \mathrm{CT}$ at different beamlines using specifications (photon energy, nominal pixel size, spatial resolution), which provided comparable data on the vessel morphology. Both contrast and spatial resolution were sufficient to extract the vessel tree down to the smallest capillaries. However, the limited photon statistics identified as noise and the presence of artifacts (cf. for example ring artifacts) are the dominating factors that restrict the precision of the derived quantities to around $10 \%$. For both healthy and cancerous tissues the vessel volume fraction obtained from the 2D analysis of tomographic slices depends on the choice of the slice. As a result, an analysis in $3 \mathrm{D}$ or of a sufficiently large number of $2 \mathrm{D}$ slices is necessary to quantify the 3D vessel tree (see Fig. 3 and Table 1). In contrast to the vessel density reported by Folarin et al. (2010), the vascularization in the present tumor is reduced with respect to the healthy one. Folarin et al., however, also stated that there is a lower vessel density in the core relative to the peripheral 
tumor samples (Folarin et al., 2010). As the vessel structure in tumor tissue appears rather unpredictable, tumors with few or no vessels have been reported as well (Bullitt et al., 2003a). Figure 2 illustrates how the phase tomogram reveals the micro-vascularization. We assume that the hemoglobin in the blood provides sufficient contrast to distinguish the vessels from the surrounding tissue. The tomography slices show vessels in the outer regions of the tumors only, but the phase contrast reveals fewer vessels in total than the corrosion casts as shown in Table 1. Histologically no endothelial cells were found in the interior of the tumor specimen. Since the tumor measured by means of phase tomography is younger ( 7 days) than the tumor used for the corrosion cast (14 days), it can be reasonably assumed that the cancerous angiogenesis is still at an early stage of its development (Naumov et al., 2006).

The 3D vectorization of the capillary networks as shown in Fig. 4 allows a quantitative assessment of the vessel characteristics. The casts of cancerous and healthy tissues were used to discover the differences between their micro-vascular structures.

The tortuosity was expected to be larger in the tumor tissue (Bullitt et al., 2003a; Bullitt et al., 2003b). The present results confirm this expectation in terms of DM and SOAM (see Table 1). However the tortuosity depends strongly on the choice of the selected region. In the present study, healthy tissue revealed tortuous vessels as well (see Fig. 5 (a) inset). According to Potter and Groom (1983) tortuous capillaries can appear in the neighborhood of a muscle due to muscle compressions enforced by the cast preparation itself. The vessels of the healthy tissue were indeed extracted from the muscular flank of a mouse. Additionally, Aharinejad et al. (1998) confirmed that capillaries with diameters below $15 \mu \mathrm{m}$ can appear tortuous in both cancerous and healthy tissues.

The mean vessel diameter (Table 1) is smaller in the cancerous vessel cast and comparable with the result of a previous study (Brey et al., 2002). This observation is in agreement with the assumption of an exceeded angiogenesis in tumors involving a dense network of capillaries (Fukumura et al., 2010; Fukumura and Jain, 2007; Fukumura and Jain, 2008). As a result, the evaluated vessel diameter can be used to distinguish between healthy and cancerous tissues (see also Fig. 6). However, the value derived from the cast and the actual value differ due to two reasons. First, the corrosion cast method may cause tissue shrinkage of several percent. Second, the corrosion of the surrounding tissue also affects the vessel wall. Hence the evaluated vessel diameter of the casts describes rather the diameter of the vessel lumen. Another problem in corrosion casting is the presence of leaked polymer (Folarin et al., 2010), particularly, in tumor tissue where the vessel walls have become damaged. Figure 7 shows characteristic features that are interpreted as a result of starting leakages through damaged vessel walls.

For that reason we present the results of the phase contrast $\mu \mathrm{CT}$ method without contrast enhancement and polymer leakages. The vessel diameters are comparable with the values of corrosion casts when the thickness of the vessel wall is taken into account. The capillary wall (including endothelium, basal lamina, and pericytes) has a thickness of approximately $1.0 \mu \mathrm{m}$ in healthy tissue and of $1.2 \mu \mathrm{m}$ in peritumoral tissue (Bertossi et al., 1997). To allow a better comparison with the phase tomography the diameter in corrosion casts have to be increased by $2 \mu \mathrm{m}$.

Figure 4 shows that the vessel tree obtained from the phase tomography data seems to include numerous disconnected vessels. The disconnectivities can be a natural phenomenon or a result of the segmentation. During the Frangi filtering noise can lead to both falsely detected and wrongly disconnected vessels. Since the vesselness is reduced at the bifurcation points, wrong disconnectivities occur particularly at the branching points. As a result the number of detected bifurcations is smaller in the phase contrast based data. The number of bifurcations in the corrosion cast, however, is overestimated due to polymer leakages. 
The mean vessel length varies slightly for different regions. The phase contrast dataset delivers the smallest value, because part of the vessels might not have been detected by the segmentation tool.

The fractal dimensions of the capillary network in cancerous and healthy tissues shows comparable values and can be explained using the 3D diffusion-limited aggregation model (Sapoval, 2001).

The presence of non-connected vessels, or blind endings, in the segmented volumes can be due either to an artifact in the image acquisition/reconstruction or to a real physiological situation. In the corrosion casted tumor measured with absorption contrast mode, such endings have to be considered as an artifact since we assume that the vessels have to be connected to allow the casting polymer to penetrate. It is therefore impossible to visualize real blind endings with this procedure. In the phase contrast tomography, however, it is possible to visualize real blind endings but unfortunately impossible to distinguish them from the artifacts. Nevertheless, from a biological point of view, the vascular structure of the tumor presents several non-connected vessels due to the continuous and non-controlled rearrangement of the vessel structure (Marme and Fusenig, 2007).

\section{Conclusion}

As tumor tissues are characterized by stronger neo-vascularization with respect to healthy tissues, the capillaries are of special interest. This study shows that for a mouse model the mean diameter of capillaries in the healthy tissue is twice as large as the one in the cancerous tissue, while there are only marginal differences concerning vessel volume fraction, tortuosity, fractal dimension, bifurcation density and angle. The mean bifurcation angle varies within the narrow range of 110 to 120 degrees.

Phase tomography is a powerful alternative for the vessel visualization as it allows imaging the vascularization without any material-based preparation procedure. In comparison to the corrosion cast, this approach enables further analyses such as optical microscopy of the histological slices. In future research the segmentation can be improved further by applying hysteresis thresholding (Wilson and Noble, 1997). It is expected to detect the bifurcation points and vascular structures in areas of weak contrast more reliably by the local choice between two thresholds. As a result, the number of vessel interrupted by the segmentation is expected to be reduced leading to a higher reliability of the quantification. From the clinical perspective, the consideration of the vascularization and the angiogenesis is important to enhance diagnosis and therapy of tumors, particularly in the brain (Jain et al., 2007). Chemotherapy of brain tumors has been limited by the capillary blood-brain barrier. Treatments, such as ultrasound enhanced delivery, are typically tested and developed on mouse and rat models (Provenzale et al., 2005; Treat et al., 2007; Yang and Horng, 2011) by studying the effect of vessel wall rupture. Thus future investigations are expected to focus more on the analysis of the capillary vascularization in murine brain tumors.

\section{Acknowledgements}

The authors gratefully acknowledge E. Meyer from the Institute of Zoology, University of Zurich for providing assistance during the corrosion cast preparation. We also thank F. Marone and M. Stampanoni for their assistance during the beam time at SLS, A. Rack for his assistance at ESRF and M. Langer (ESRF) as well as F. Beckmann (HZG) for their help in data reconstruction. The project was financially supported by Swiss National Science Foundation (200021_127297/1). This research was also supported by the NCCR Co-Me (Computer-Aided and Image-Guided Medical Interventions) of the Swiss National Science Foundation (http://www.co-me.ch). One of the authors (T.W.) acknowledges support from RTRA 'Digiteo' and RTRA 'Triangle de la Physique' (grants 2009-034T and 2009-79D). Beamtime was granted through the proposals II0080040-EC (HASYLAB at DESY, Hamburg, 
Germany), MD-498 (ESRF, Grenoble, France) and e12159 (SLS at PSI, Villigen, Switzerland). 


\section{Captions of Tables and Figures}

Table 1

Vessel parameters for the cast of healthy tissue (nominal pixel size: $1.98 \mu \mathrm{m}$ ) and of cancerous tissue (nominal pixel size: $0.74 \mu \mathrm{m}$ ) as well as the vessels derived from phase tomography of a tumor (nominal pixel size: $1.5 \mu \mathrm{m}$ ). The parameters were determined for the region $\mathrm{ROI}_{2}$ with $590 \times 590 \times 500$ voxels.

Fig. 1. Vessel tree scheme explaining the parameters used for the quantification of capillary networks. The vessels in healthy and cancerous tissue are characterized by their length $L$, the bifurcation angle $\gamma_{B I F}$, and the diameter $D m=2 R m$, where $R m$ denotes the vessel radius.

Fig. 2. (a) The tomography slice of a tumor measured with phase contrast $\mu \mathrm{CT}$ (beamline ID19, ESRF, France) displays vessels down to the capillary size. (b) The zoom-in shows lipid cells (black) and vessels (white). The gray scale is based on the decrement $\delta$ of the X-ray refractive index. (c) The blood vessel segmentation was achieved using Frangi filtering of the tomography slices neglecting noise. The zoom-in (b) is also shown in (c) as gray-scale plane.

Fig. 3. The 3D distance transform of the casted cancer tissue $\left(\mathrm{ROI}_{1}\right)$ reveals the void distances between the vessels. The void distance is evaluated on the segmented voxel data set in a robust manner, by determining the distance of a voxel to its closest neighbor vessel in 3D. The mean void distance relates to the mean distances between the vessels and therefore inversely correlates to the vessel density.

Fig. 4. The 3D representations of the region $\mathrm{ROI}_{1}$ of each dataset (left) and the derived vectorized vessel trees (right). The images correspond to the cast of healthy vessels (a), the cast of the tumor (b) and the vessels from the tumor in PFA acquired by phase tomography (c). The red-colored dots in the vectorized representation indicate the bifurcation points.

Fig. 5. The images show characteristic 3D representations of vessel casts of the dense capillary network from healthy tissue (a) with zoom-in (b) and from cancerous tissue (c) with zoom-in (d). The dashed ellipses highlight twisted vessels, present in the healthy and cancerous tissues, which were quantified using tortuosity.

Fig. 6. Vessel diameter distribution in the cancerous and healthy tissues investigated. The vessel diameters of the cast from healthy tissue are twice as larger as the ones from the cancerous tissue.

Fig. 7. In this region of the cast, prepared from the tumor, a significant number of polymer leakages were found, especially in the region highlighted in the dashed ellipse. Here, the polymer presumably penetrated damaged vessel walls during cast preparation. Damaged vessel walls are characteristic of cancerous tissues in advanced stages. Such preparation artifacts influence the quantitative analysis of the vessel tree. Therefore, casting can only be applied successfully for healthy tissues or cancerous tissues in early stages. 


\section{References}

Aharinejad, S., et al., 1998. Morphometry of human coronary arterial trees. Anat. Rec. 251, 50-59.

Beckmann, F., 2002. Microtomography using synchrotron radiation as a user experiment at beamlines BW2 and BW5 of HASYLAB at DESY. Proc. SPIE 4503, 34-42.

Beckmann, F., et al., 2008, High density resolution in synchrotron-radiation-based attenuation-contrast microtomography. Proc. SPIE 7078, 70781D.

Bertossi, M., et al., 1997. Ultrastructural and morphometric investigation of human brain capillaries in normal and peritumoral tissues. Ultrastruct. Pathol. 21, 41-49.

Brey, E. M., et al., 2002. A technique for quantitative three-dimensional analysis of microvascular structure. Microvasc. Res. 63, 279-294.

Bullitt, E., et al., 2003a. Vascular attributes and malignant brain tumors. Med. Image Comput. Comput. Assist. Interv. 2003, 671-679.

Bullitt, E., et al., 2003b. Measuring tortuosity of the intracerebal vasculature from MRA images. IEEE Trans. Med. Imag. 22, 1163-1171.

Cloetens, P., et al., 1999. Holotomography: Quantitative phase tomography with micrometer resolution using hard synchrotron radiation x rays. Appl. Phys. Lett. 75, 2912-2914.

Collins, J., et al., 2011. Assessment of vascular network segmentation. Int. J. Image Processing 4, 584-599.

Cornea, N. D., et al., 2005. Computing hierarchical curve-skeletons of 3D objects. Vis. Comput. 21, 945-955.

Di Ieva, A., et al., 2011. Angioarchitectural heterogeneity in human glioblastoma multiforme: A fractal-based histopathological assessment. Microvasc. Res. 81, 222-230.

Folarin, A. A., et al., 2010. Three-dimensional analysis of tumour vascular corrosion cast using stereroimaging and micro-computed tomography. Microvasc. Res. 80, 89-98.

Frangi, A. F., et al., 1998. Multiscale vessel enhancement filtering. Med. Image Comput. Comput. Assist. Interv. 1496, 130-137.

Fukumura, D., et al., 2010. Tumor microvasculature and microenvironment: Novel insights through intravital imaging in pre-clinical models. Microcirculation 17, 206-225.

Fukumura, D., Jain, R. K., 2007. Tumor microvasculature and microenvironment: Targets for anti-angiogenesis and normalization. Microvasc. Res. 74, 72-84.

Fukumura, D., Jain, R. K., 2008. Imaging angiogenesis and the microenvironment. Apmis $116,695-715$.

Guigay, J.-P., et al., 2007. Mixed transfer function and transport of intensity approach for phase retrieval in the fresnel region. Optics Lett. 32, 1617-1619.

Heinzer, S., et al., 2006. Hierarchical microimaging for multiscale analysis of large vascular networks. Neuroimage 32, 626-636.

Jain, R. K., et al., 2007. Angiogenesis in brain tumours. Nature Rev. Neurosci. 8, 610-622.

Kak, A. C., Slaney, M., 1988. Principles of Computerized Tomographic Imaging. IEEE Service Center, Piscataway, NJ.

Kroon, D.-J., http://www.mathworks.com/matlabcentral/fileexchange/24409-hessian-basedfrangi-vesselness-filter. Hessian Based Frangi Vesselness Filter 2009.

Krucker, T., et al., 2006. New polyurethane-based material for vascular corrosion casting with improved physical and imaging characteristics. Microsc. Res. Techn. 69, 138-147.

Lang, S., et al., 2012. Global and local hard X-ray tomography of a centimetre-sized tumor vessel tree. J. Synchr. Rad. 19, 114-125.

Langer, M., et al., 2008. Quantitative comparison of direct phase retrieval algorithms in inline phase tomography. J. Med. Phys. 35, 4556-4566.

Langer, M., et al., 2010. Regularization of phase retrieval with phase- attenuation duality prior for 3-D holotomography. IEEE Trans. Image Process. 19, 2428-2436. 
Lesage, D., et al., 2009. A review of 3D vessel lumen segmentation techniques: models, features and extraction schemes. Med. Image Anal. 13, 819-845.

Lloyd, B. A., et al., 2007. A coupled finite element model of tumor growth and vascularization. Med. Image Comput. Comput. Assist. Interv. 10, 874-881.

Mancardi, D., et al., 2008. Fractal parameters and vascular networks: Facts \& artifacts. Theor. Biol. Med. Model. 5, 1-12.

Mandelbrot, B., 1982. The Fractal Geometry of Nature. W. H. Freeman, San Francisco.

Marme, D., Fusenig, N. (Eds.), 2007. Tumor angiogenesis - basic mechanisms and cancer therapy. Springer, Berlin.

Müller, B., et al., 2007. Morphology of bony tissues and implants uncovered by highresolution tomographic imaging. Int. J. Mat. Res. 98, 613-621.

Müller, B., et al., 2006a. Blood vessel staining in the myocardium for 3D visualization down to the smallest capillaries. Nuclear Instr. Meth. Phys. Res. 246, 254-261.

Müller, B., et al., 2006b. Three-dimensional assessment of brain tissue morphology. Proc. SPIE 6318, 631803.

Müller, B., et al., 2008. High-resolution tomographic imaging of microvessels. Proc. SPIE 7078, 70780B.

Müller, B., et al., 2002, Non-destructive three-dimensional evaluation of biocompatible materials by microtomography using synchrotron radiation. Proc. SPIE 4503, 178-188.

Müller, B., et al., 2002b, Non-destructive three-dimensional evaluation of a polymer sponge by micro-tomography using synchrotron radiation. Biomol. Eng. 19, 73-78

Naumov, G. N., et al., 2006. Role of angiogenesis in human tumor dormancy: Animal models of the angiogenic switch. Cell Cycle 5, 1779.

Plouraboué, F., et al., 2004. X-ray high-resolution vascular network imaging. J. Microsc. 215, 139-148.

Potter, R. F., Groom, A. C., 1983. Capillary diameter and geometry in cardiac and skeletal muscle studied by means of corrosion casts. Microvasc. Res. 25, 68-84.

Provenzale, J. M., et al., 2005. The role of blood-brain barrier permeability in brain tumor imaging and therapeutics. Am. J. Roentgenology 185, 763-767.

Rijken, P. F., et al., 1995. Application of an image analysis system to the quantification of tumor perfusion and vascularity in human glioma xenografts. Microvasc. Res. 50, $141-153$.

Sapoval, B., 2001. Universalités et fractales. Collection Champs, Flammarion.

Sato, Y., et al., 1998. Three-dimensional multi-scale line filter for segmentation and visualization of curvilinear structures in medical images. Med. Image Anal. 2, 143168.

Stampanoni, M., et al., 2006. Trends in synchrotron-based tomographic imaging: the SLS experience. Proc. SPIE 6318, 63180M.

Treat, L. H., et al., 2007. Targeted delivery of doxorubicin to the rat brain at therapeutic levels using MRI-guided focused ultrasound. Int. J. Cancer 121, 901-907.

Weitkamp, T., et al., 2012. Status and evolution of the ESRF beamline ID19. AIP Conf. Proc. 1221, 33-38.

Wild, R., et al., 2000. Quantitative assessement of angiogenesis and tumor vessel architecture by computer-assistend digital image analysis: Effects of VEGF-toxin conjugate on tumor microvessel density. Microvasc. Res. 59, 368-376.

Wilson, D., Noble, J., 1997. Segmentation of cerebral vessels and aneurysms from MR angiography data. Inform. Proc. Med. Imag. 1230, 423-428.

$\mathrm{Wu}, \mathrm{X}$., et al., 2008. Quantitative analysis of the microvasculature growing in the fibrin interface between a skin graft and the recipient site. Microvasc. Res. 75, 119-129. 
Yang, F.-Y., Horng, S.-C., 2011. Ultrasound enhanced delivery of macromolecular agents in brain tumor rat model. Proc of Ann. Int. Conf. IEEE Eng. Med. Biol. Soc. 2011, 5573-5576.

Zabler, S., et al., 2005. Optimizing of phase contrast imaging using hard X rays. Rev. Sci. Instrum. 76, 1-7.

Zhou, J., et al., Vessel boundary extraction using ridge scan-conversion deformable model Int. Symp. on Biomed. Imaging: Nano to Macro, Arlington, VA 2006, pp. 189-192. 